\title{
Medical Therapy for Crohn's Disease: Top-Down or Step-Up?
}

\author{
Filip Baert $^{\mathrm{a}}$ Renzo Caprilli ${ }^{\mathrm{b}}$ Erika Angelucci ${ }^{\mathrm{b}}$ \\ Departments of Gastroenterology, a Hospital of Leuven, Belgium, and 'University of Rome 'La Sapienza', \\ Rome, Italy
}

\section{Key Words}

Crohn's disease, medical therapy · Crohn's disease

treatment, pros and cons - Step-up vs. top-down strategy about long-term safety and the high costs of infliximab are further factors supporting a more careful approach to the management of $C D$.

Copyright $\odot 2007$ S. Karger AG, Basel

\begin{abstract}
The emergency of effective biological therapy in the treatment of Crohn's disease (CD) has led to a clinical debate about 'step-up versus top-down strategy'. Step-up refers to the classic therapeutic approach, namely progressive intensification of treatment as disease severity increases. Topdown refers to the early introduction, in all CD patients, of intensive therapies, including biological agents and immunosuppressive drugs, with the aim of avoiding complications and improving quality of life, starting from the assumption that these drugs may interfere with the natural history of the disease. Very recently the Belgian IBD research group together with the Gut Club of North Holland designed 'the Step Up versus Top Down Trial'. Combination of infliximab with immunosuppressives at onset was better than the current standard approach in terms of both induction and maintenance of remission. However, several observations still limit the use of infliximab as first-line treatment in adult $C D$ patients. In particular, the epidemiological observation that over $50 \%$ of $C D$ patients have a mild disease over time and will never require aggressive therapies is against the indiscriminate use of top-down strategy. Lack of markers able to identify high-risk patients, discussions
\end{abstract}

\section{Arguments for: F. Baert (Belgium)}

As physicians we have to be honest about what we have reached in the past in our patients with inflammatory bowel disease (IBD). Yet, oftentimes we have been able to improve symptoms, more rarely patients became free of symptoms. But unfortunately this was often achieved at the cost of side effects of the medications. Keeping the patients in a maintained remission was already rather exceptional. And up until now very recently achieving tissue healing of the mucosa of the bowel was impossible. However, tissue healing seems to be important in order to avoid the known and frequent complications such as fistulae, abscesses, adhesions, strictures and obstructions. Therefore, tissue healing may be a goal we should aim for in the treatment of Crohn's disease (CD). A cure is of course a dream far away for every patient being diagnosed with a chronic IBD. CD puts a considerable burden on society in terms of quality of life of our patients but also costwise with surgery and hospitalization for flare-ups accounting for $80 \%$ of the costs [1]. This does not include the additional cost of sick leave and disability.

\section{KARGER}

Fax +4161306 1234 E-Mail karger@karger.ch www.karger.com (c) 2007 S. Karger AG, Basel

0257-2753/07/0253-0260\$23.50/0

Accessible online at:

www.karger.com/ddi
Renzo Caprilli, MD

Policlinico Umberto I, Viale del Policlinico 155

IT-00161 Rome (Italy)

Tel. +39064460 009, Fax +39064463737

E-Mail renzo.caprilli@uniroma1.it 
With all these thoughts in mind, the Belgian IBD research group together with the Gut Club of North Holland designed 'the Step-Up versus Top-Down Trial' [2]. The idea in 'the Step-Up versus Top-Down trial' was to compare the current standard treatment (step-up therapy) with a more aggressive top-down (TD) strategy. The reason being that induction therapy with steroids is only moderately effective and associated with significant toxicity. Infliximab, which is currently restricted for refractory $\mathrm{CD}$, is a very effective induction therapy. In addition, sustained clinical benefit could possibly be obtained by adding azathioprine or methotrexate right from the start. Therefore, in the TD arm, patients were randomized to three inductions infusions of infliximab (weeks 0,2 and 6) in addition to azathioprine $(2.5 \mathrm{mg} / \mathrm{kg}$ b.w.). If patients relapsed after the first three induction doses, a new infliximab infusion could be given episodically upon every relapse. Steroids were reserved only as a last resort for patients refractory to the combination of infliximab with azathioprine.

In the step-up strategy (SU) patients were treated with steroids with a fixed tapering regimen when patients relapsed they had a second course of steroids and when failing again or when resistant to steroids azathioprine was added. When patients failed 3 months of azathioprine (either because of acute relapse or when chronically active), infliximab was added as third-line therapy on an episodic basis after a three-dose induction. In both arms, patients were switched to methotrexate in case of azathioprine allergy of intolerance.

The primary endpoints of study were: (1) Is aggressive medical therapy, both induction and maintenance, more efficacious than standard therapy, in recent onset CD? (2) Can early introduction of immunomodulators modify the clinical course of recent onset CD? (3) Can CD be treated without steroids?

The study was an open-label multicenter study conducted in 26 centers in Belgium and The Netherlands and 1 center in Germany. To be included, patients had to be newly diagnosed $\mathrm{CD}$, not being treated with steroids or immunomodulators in the past. In total, 129 patients are randomized, 64 patients in the SU group and 65 patients in the TD group. Both groups were equally balanced in terms of disease location, gender, age, weight, smoking behavior and CDAI at baseline. Patients were moderately to severely active (mean CDAI TD 331 and 306 in the SU group).

In terms of the primary outcome at months 6 and 12, both treatment strategies were comparable with a $75 \%$ $\mathrm{TD}$ remission rate compared to a $73 \% \mathrm{SU}$ remission rate

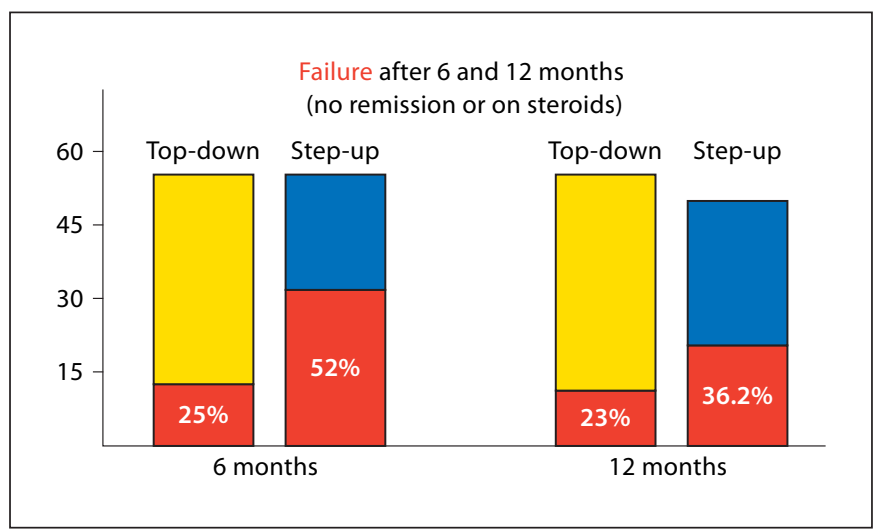

Fig. 1. Step-up vs. top-down trial.

at 6 months, and a $77 \%$ TD remission rate compared to a $67 \%$ SU remission rate at 12 months. When we reanalyzed the data using the more stringent definition of treatment success (that is being in remission without being on steroids) the results looked quite different. In the TD group, $75 \%$ patients were in remission at month 6 compared to $48 \%$ of patients in the SU group that were in remission and not on steroids; at 12 months, treatment success was reached in 77 and $47 \%$ of patients, respectively (fig. 1). When looking at the time frame in which the remission was obtained, TD patients reached their remission much earlier on, with already $60 \%$ of patients being in remission at week 10 .

The study also included an endoscopic arm. Patients were invited to have an ileocolonoscopy 2 years after randomization. The endoscopies were scored with the SES (simple endoscopy score) [3]. All results were recorded and were compared with the baseline result by a single investigator of course blinded for the treatment arm. The lesions were scored in 5 sections raging from 0 (no ulcers) to 3 (ulcerated stenosis). The SES score diminished to an average of $3.1( \pm 2.9)$ in the SU group compared to 0.7 $( \pm 1.5)$ in the TD group $(\mathrm{p}<0.0001)$. Looking at it in a different way there was a complete ulcer disappearance in 15 out of the $20 \mathrm{TD}$ patients (57\%) compared to 3 of 14 patients in the SU group (21\%) ( $p=0.003)$. In terms of safety there was no difference in both treatment strategies.

This study led us to the following conclusions for the step-up (current approach): (1) Steroids are of limited clinical benefit for induction of remission. (2) Repeat steroids and early introduction of immunosuppressives are required to achieve clinical remission. (3) Almost two- 


\section{Color illustration online only!}

Fig. 2. Infliximab + azathioprine in steroid-resistant patients [from Lemann et al: Gut 2003;52(suppl 6):A44-A45].

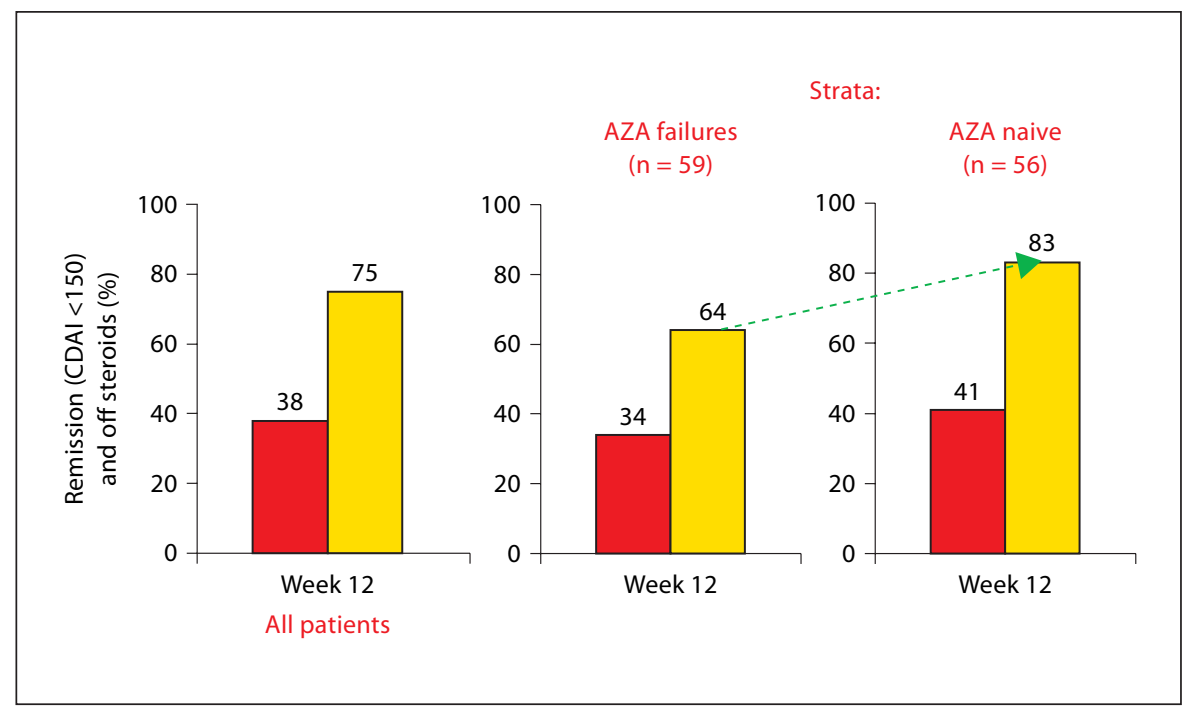

thirds of patients will require immunosuppressives within 1 year to maintain remission. (4) Mucosal healing is not achieved in the majority of patients.

Combination of infliximab with immunosuppressives at onset is superior to the current standard approach both in terms of induction of remission and maintenance of remission. This strategy enables to completely avoid steroids and leads to long-term mucosal healing in $57 \%$ of patients.

Regarding the use of steroids and the risks of infliximab, interesting data are emerging from the TREAT Registry [4]. Multivariant analysis of the TREAT Registry data covering $>10,000$ patient-years of observation show that only the use of corticosteroids and not the use of infliximab or immunosuppressants increase the risk for mortality or serious infections. So avoiding the use of steroids by using a more aggressive (TD) strategy may be safer compared to the SU strategy. Other data supporting the use of infliximab are data coming from the ACCENT trials showing that maintaining therapy by infliximab leads to significantly fewer hospitalizations or surgeries compared to placebo maintenance [5]. These data are to be compared with historical controls showing that despite the earlier and more widespread use of immunosuppressants in the last decade, the cumulative need for intestinal resection in different cohorts of patients has not been reduced with a stable percentage of about $30 \%$ of patients needing surgery in the first 6 years after diagnosis [6].

Other data in favor of early use of TNF blockers come from the GETAID trial [7]. Lemann et al. examined pro- spectively a group of patients that were steroid-dependent where infliximab or placebo infusions were added together with azathioprine. In the azathioprine-naive patients, $83 \%$ had a response compared to only $64 \%$ in the azathioprine-failing patients (fig. 2). Further evidence for earlier and better treatment comes from the pediatric trial group. The most spectacular data come from the Markowitz trial [8] showing an almost $100 \%$ response in early diagnosed $C D$ patients treated with 6-mercaptopurine as maintenance therapy from the start. This response is being maintained throughout the first 2 years of followup. Another pediatric trial by Kugathasan et al. [9] shows a clearly better and longer response in children with early-onset CD compared to later-onset CD patients.

A possible downside of earlier infliximab therapy may be the increased cost. This increased cost leads to less surgery and fewer hospitalizations. This may compensate possibly for the extra costs in medications. A first cost simulation assessment was made by Jewell et al. [10] using retrospective data from seven centers in the UK. They concluded that infliximab appeared cost-effective for selected patients based on the savings made by the reduced number of surgeries, diagnostic procedures and inpatients stays over a 6-month period.

Of course, future trials will need to demonstrate which patients are in need for aggressive therapy early on compared to patients with a more indolent course of their disease. A lot of research focuses on trying to identify subgroups of patients using different markers including serologic, genetic and just clinical features. However, for the moment we lack specific reliable markers. If inflix- 
Color illustration online only!

Fig. 3. Mild disease/long-term remission [from 11].

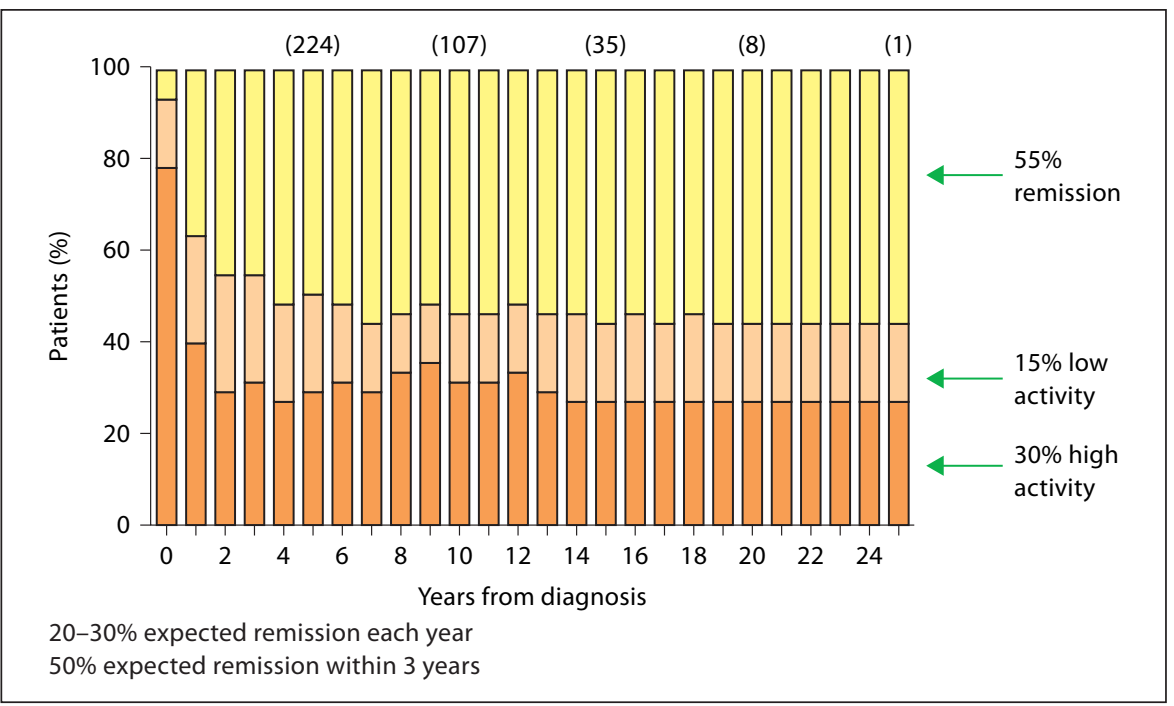

imab and all the other biological agents are now being used both for induction of remission and for maintenance of remission in a maintenance manner, one can argue whether we need concomitant immunosuppressives. The SONIC trial, being conducted throughout the world, which randomizes early $\mathrm{CD}$ patients in three arms - azathioprine alone, infliximab + azathioprine or infliximab alone - will probably bring the answer.

As a general conclusion it is fair to say that immunomodulators and biological agents now offer the potential to modify the disease early in its course. Steroids may be deleterious, offer no (long-term) benefit and can be avoided. Early aggressive therapy including infliximab enables us to better achieve our treatment goals: rapid control of symptoms, maintenance of remission, reduction of surgeries and hospitalizations and improvement in patient quality of life.

\section{Arguments against: R. Caprilli and E. Angelucci (Italy)}

The emergency of effective biological therapy in the treatment of $\mathrm{CD}$ has led to a clinical debate as far as the so-called question of SU vs. TD strategy is concerned. SU refers to the classic therapeutic approach resulting in progressive intensification of therapies with the increasing severity of the disease. TD, on the other hand, refers to the early introduction, in every CD patient, of intensive therapies, including biological agents and immunosuppressive drugs, with the aim of avoiding complications and improving quality of life, starting from the assump- tions that these drugs may interfere with the natural history of the disease.

However, to date, several clinical and epidemiological observations still limit the use of infliximab as first-line treatment in adult CD patients.

(1) Data from epidemiological studies and mathematical models: Data from a large population-based study performed in Copenhagen County, Denmark, showed that $80 \%$ of CD patients maintain high disease activity in the first year after diagnosis. This percentage decreases dramatically after the first year of disease history, with about $55 \%$ of CD patients in stable remission at any given year [11] (fig. 3). Furthermore, two different population-based studies from Copenhagen County, Denmark, and Olmsted County, Minn., USA, showed that only $43-56 \%$ of CD patients need steroid therapy $[12,13]$. Even data from the Markov model analysis showed that most (about 65\%) CD patients' lifetimes are spent in remission both medically and surgically induced [14]. Lastly, it has recently been found that about half of CD patients already have a complicated disease (both structuring and penetrating) at diagnosis [15]. In these cases, biological therapies would have no chance to modify the natural history of the disease, even if used early.

Taken together, these data indicate that the indiscriminate use of infliximab as first-line therapy would represent an overtreatment for most of CD patients.

(2) Lack of markers able of identifying high-risk groups of patients: Several approaches have been explored to identify biomarkers, both serological and genetic, able of predicting the $\mathrm{CD}$ course. Among serological mark- 


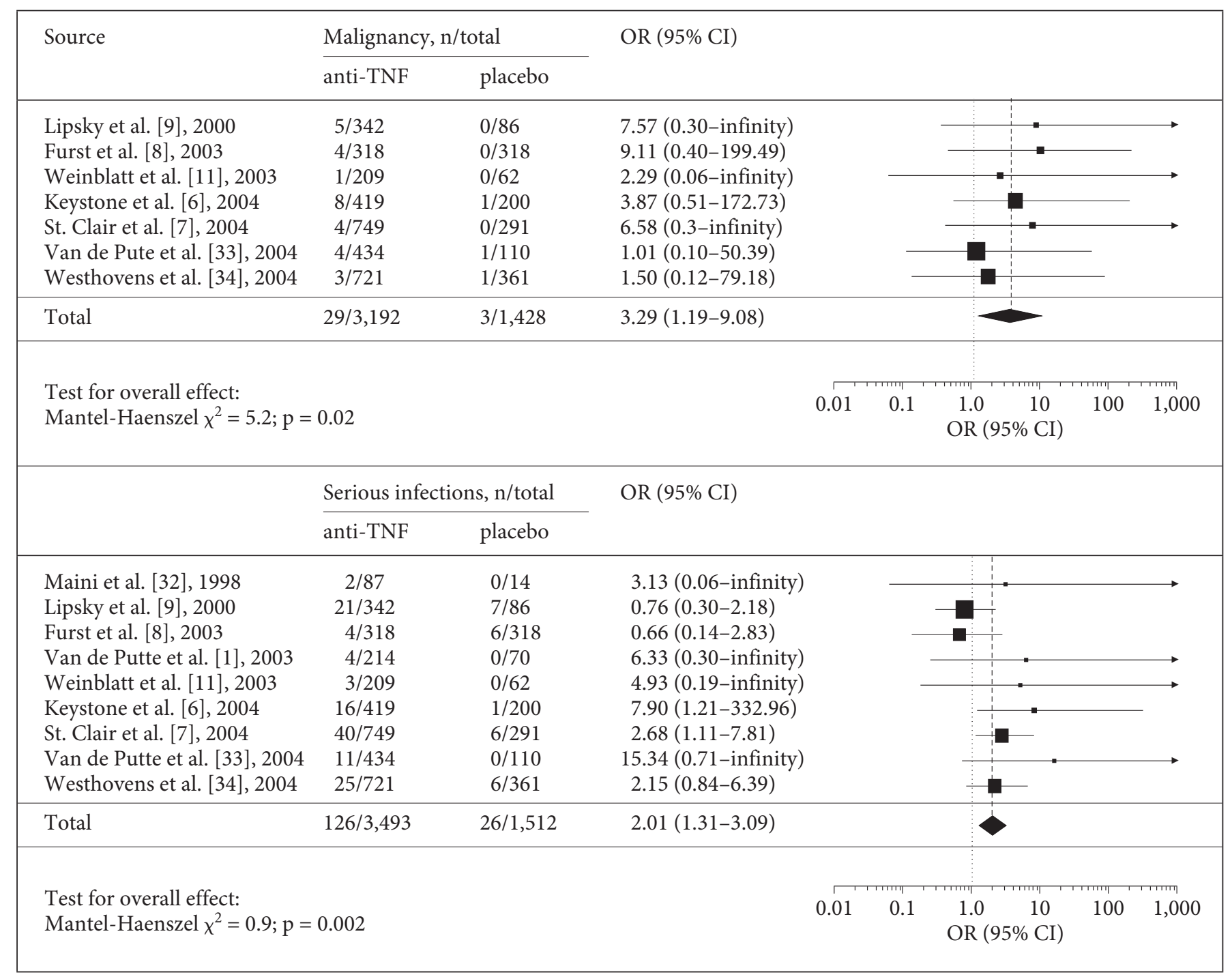

Fig. 4. Long-term safety of TNF inhibitors in rheumatoid arthritis - a meta-analysis [from 20].

ers, perinuclear antineutrophil cytoplasmic antibodies (pANCA) and anti-Saccharomyces cerevisiae antibodies (ASCA) are the most studied. New markers have recently been introduced, such as anti-chitobioside antibodies (ACCA), anti-laminaribioside antibodies (ALCA), antibodies to Escherichia coli outer membrane porin (anti$\mathrm{OmpC} \mathrm{Ab}$ ), antibodies to the Pseudomonas fluorescensassociated sequence I2 (anti-I2 Ab) and antibodies to CBirl flagellin; their sensitivity and predictive value still remain to be defined [16]. Also, polymorphisms of innate immunity genes such as NOD2/CARD15 and Toll-like receptors (TLRs) are not sufficient to diagnose CD or to predict its course.
To date, all these markers are not yet able to guide therapeutic choices, by identifying CD patients who would need aggressive early treatment.

(3) Lack of robust clinical data supporting top-down strategy: To date, with the exception of the already mentioned trial of the Belgian IBD research group together with the Gut Club of North Holland, there are no controlled clinical studies supporting the superiority of TD strategy with respect to $\mathrm{SU}$ in the treatment of adult $\mathrm{CD}$ patients [2]. However, preliminary studies indicate that the TD approach may be advantageous in children $[8$, 9]. 
(4) Long-term safety: The main drawback of early use of infliximab remains the strong and prolonged immunosuppression that may lead to severe toxicity and immunogenicity. In fact it has clearly been shown that infliximab is not only able to bind soluble TNF, but also to induce apoptosis of immune cells expressing TNF at their surface. Infliximab should therefore not be considered an immunomodulator but an immunosuppressive drug.

Data from different settings, including RCTs and cohort studies, substantially indicate that $C D$ patients treated with infliximab do not have an excess of mortality $(0.4$ vs. $1.2-1.3 \%$, respectively) and neoplasia, including lymphomas, but incidence of serious infections is slightly increased (4-4.2 vs. $8.2-8.3 \%$, respectively) [17]. In particular the use of TNF inhibitors is associated with a six fold increased risk of active tuberculosis [18]. Mortality of tuberculosis in the early days of its recognition in association with infliximab use is about $10 \%$ [18]. The high prevalence of anergy to standard tuberculin skin test among $\mathrm{CD}$ patients contributes to make potentially ineffective screening for tuberculosis before anti-TNF therapies $[18$, 19].

Data from the TREAT (Therapy Resource, Evaluation and Assessment Tool) registry, evaluating long-term safety in $>6,000 \mathrm{CD}$ patients, have also shown an increased risk of serious infections in $\mathrm{CD}$ patients treated with TNF inhibitors, even if this risk seems to be due to previous use of steroids, according to Cox hazard analysis [4]. However, a very recent meta-analysis on the safety of TNF inhibitors in rheumatoid arthritis patients has shown a significantly increased risk of malignancies (OR 3.29; CI 95\% 1.09-9.08) and serious infections (OR 2.01; CI 95\% 1.31-3.09). Risks were also higher in patients treated with doses and schedules similar to those used in CD patients [20] (fig. 4).
(5) Costs. It has been demonstrated that in fistulizing CD patients, infliximab is able to reduce hospitalizations and surgery [5], which represent about $80 \%$ of costs for $\mathrm{CD}$ [1]. However, over $50 \%$ of CD patients maintain stable remission or mild disease activity over time, and therefore do not need hospitalization and surgery at all [11]. From this point of view, the advantage coming from TD therapy seems to be small. Anyway the high direct costs of infliximab (in Italy for a 70-kg patient, induction therapy costs about EUR 8,400 and 1-year maintenance EUR 18,500) strongly limit its use. Even in patients with rheumatoid arthritis, in whom the early treatment with TNF- $\alpha$ inhibitors had clearly shown the regression of lesions, TD strategy is not generally applied. In fact, to limit medical expenses, several European rheumatologic societies recommend the use of TNF- $\alpha$ inhibitors only after failure of an adequate trial of another effective diseasemodifying anti-rheumatic drug (DMARD), including methotrexate [21].

\section{Conclusions}

In conclusion, there is convincing evidence that biological therapy is very effective for moderately to severely active CD patients, but it remains to be demonstrated whether such CD patients exist in whom the benefit of early intensive therapy with biological agents outweighs the long-term risk. To date, there is no sufficient evidence to accept an indiscriminate inversion of therapeutic pyramid in adult CD patients. Future identification of markers capable of predicting patients who would benefit from TD therapy could modify the current therapeutic approach.

\section{References}

1 Hay AR, Hay JW: Inflammatory bowel disease: medical cost algorithms. J Clin Gastroenterol 1992;14:318-327.

2 Hommes D, Baert F, Van Assche G, et al: A randomized controlled trial evaluating the ideal medical management for Crohn's disease: top-down versus step-up strategies. Gastroenterology 2005;128(suppl 2):A577.

-3 Daperno M, D'Haens G, Van Assche G, et al: Development and validation of a new, simplified endoscopic activity score for Crohn's disease: the SES-CD. Gastrointest Endosc 2004;60:505-512.
4 Lichtenstein GR, Feagan BG, Cohen RD, et al: Serious infections and mortality in association with therapies for Crohn's disease: treat registry. Clin Gastroenterol Hepatol 2006;4:621-630.

5 Lichtenstein GR, Yan S, Bala M, et al: Infliximab maintenance treatment reduces hospitalizations, surgeries, and procedures in fistulizing Crohn's disease. Gastroenterology 2005;128:862-869.
-6 Cosnes J, Nion-Larmurier I, Beaugerie L, et al: Impact of the increasing use of immunosuppressants in Crohn's disease on the need for intestinal surgery. Gut 2005;54:237-241.

7 Lemann M, Mary JY, Duclos B, et al (GETAID): Infliximab plus azathioprine for steroid dependent Crohn's disease patients: a randomised placebo-controlled trial. Gastroenterology 2006;130:1054-1061.

-8 Markowitz J, Grancher K, Kohn N, et al: A multicenter trial of 6-mercaptopurine and prednisone in children with newly diagnosed Crohn's disease. Gastroenterology 2000;119:895-902. 
9 Kugathasan S, Werlin SL, Martinez A, et al: Prolonged duration of response to infliximab in early but not late pediatric Crohn's disease. Am J Gastroenterol 2000;95:31893194.

10 Jewell DP, Satsangi J, Lobo A, et al: Inflix imab use in Crohn's disease: impact on health care resources in the UK. Eur J Gastroenterol Hepatol 2007; 17:1047-1052.

-11 Munkholm P, Langholz E, Davidsen M, et al: Disease activity courses in a regional cohort of Crohn's disease patients. Scand J Gastroenterol 1995;30:699-706.

12 Munkholm P, Langholz E, Davidsen M, et al: Frequency of glucocorticoid resistance and dependency in Crohn's disease. Gut 1994;35: 360-362.
13 Faubion WA Jr, Loftus EV Jr, Harmsen WS, et al: The natural history of corticosteroid therapy for inflammatory bowel disease: a population-based study. Gastroenterology 2001;121:255-260.

14 Silverstein MD, Loftus EV, Sandborn WJ, et al: Clinical course and costs of care for Crohn's disease: Markov model analysis of a population-based cohort. Gastroenterology 1999;117:49-57.

15 Papi C, Festa V, Fagnani C, et al: Evolution of clinical behaviour in Crohn's disease: predictive factors of penetrating complications. Dig Liver Dis 2005;37:247-253.

16 Abreu MT: Serologies in Crohn's disease: Can we change the gray zone to black and white? Gastroenterology 2006;131:664-666.

-17 Sandborn WJ, Loftus EV: Balancing the risks and benefits of infliximab in the treatment of inflammatory bowel diseases. Gut 2004; 53:780-782.
18 Keane J, Gershon S, Wise RP, et al: Tuberculosis associated with infliximab, a tumor necrosis factor- $\alpha$-neutralizing agent. $\mathrm{N}$ Engl J Med 2001;345:1098-1104.

19 Rampton DS: Preventing TB in patients with Crohn's disease needing infliximab or other anti-TNF therapy. Gut 2005,54:1360-1362.

20 Bongartz T, Sutton AJ, Sweeting MJ, et al: Anti-TNF antibody therapy in rheumatoid arthritis and the risk of serious infections and malignancies: systematic review and meta-analysis of rare harmful effects in randomized controlled trials. JAMA 2006;295: 2275-2285.

21 Valesini G, Montecucco C, Cutolo M: Recommendations for the use of biologic (TNF$\alpha$-blocking) agents in the treatment of rheumatoid arthritis in Italy. Clin Exp Rheumatol 2006;24:413-423. 